different platforms with central updating. The application has been evaluated and tested through literature search, internal validation exercises, code testing, risk analysis, and usability assessments. Usability assessments $(n=7)$ has shown mean user subjective satisfaction of 8.5 out of 10 . A screenshot from the application. Plans for post-production maintenance and surveillance have been established. A technical file for the application has been written according to Medical Devices Directive (MDD) and all other relevant harmonised standards. The process of registering the application with the MHRA and for CE marking is underway.

Conclusions The application Predict GI Cancer in IDA generates an estimate of GI cancer risk (with 95\% confidence interval), following the insertion of data for the four key variables. The whole process takes just a few seconds, which lends itself to use in busy clinical settings. Legal notices, contact system and all the supportive information for the application such as description of the population, intended users, safety information have been embedded within the application interface.

\section{IMPLEMENTATION OF SYSTEMATIC LYNCH SYNDROME TESTING IN COLORECTAL CANCER: OUTCOMES FROM A PILOT PATHWAY}

${ }^{1}$ Paul Collins*, ${ }^{1}$ Tim Andrews, ${ }^{1}$ Emma Atkinson, ${ }^{1}$ Kerrie Davis, ${ }^{2}$ Lynn Greenhalgh,

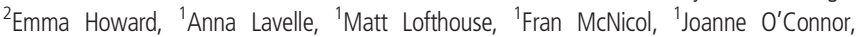
${ }^{1}$ Rachel Quinn, ${ }^{1}$ Alistair Reid, ${ }^{1}$ Paul Skaife, ${ }^{1}$ Fiona Campbell. 'Liverpool University Hospitals NHS Foundation Trust, Liverpool, UK; ' ${ }^{2}$ Liverpool Womens Hospital, Liverpool, UK

\subsection{6/gutjnl-2020-bsgcampus.68}

Introduction Lynch syndrome (LS) is an inherited genetic condition that accounts for $3.3 \%$ of colorectal tumours. Patients with LS are at risk of developing other cancers including cancer of the endometrium and urinary tract. The diagnosis of LS provides an opportunity to enrol affected patients into preventative surveillance programmes and also the opportunity to offer screening to relatives. Historically, targeted testing of patients with colorectal cancer (CRC) based on age $(<50$ years) and family history has been widely adopted into common practice.

In 2017, NICE issued a recommendation for systematic testing for LS in all patients. Implementation of the guidelines poses some organisational challenges. Consent for genetic testing must be incorporated into patient pathways for those diagnosed with CRC. Co-ordinated communication between CRC MDTs and genetics laboratories is also required.

Methods A pilot pathway for LS testing was rolled out across two UK tertiary centres. Five CRC specialist nurses underwent training to consent patients for LS testing by members of the regional Clinical Genetics team. Consent was incorporated into their standard clinic review following the initial diagnosis of CRC.

LS testing was undertaken using an immunohistochemistry 4-panel test for MLH1, MSH2, MSH6 and PMS2, with sequential BRAF V600E and MLH1 promotor hypermethylation testing in MLH1 IHC negative patients.

Results 189/196 (97\%) patients consented to LS testing. 29/ 189 (15\%) had abnormal IHC (potential LS patients). 6 cases of LS were confirmed on IHC alone (MSH2, n=1; MSH2 \& MSH6, $n=3$; MSH6, $n=1$; PMS2, $n=1$ ). A further 6 cases were identified from the remaining patients.
Overall, 12 patients $(6.3 \%$ of the tested cohort) had LS. 3 patients were $<50$ years old.

No adjustment to clinic numbers was required to accommodate consent for testing.

Conclusion Systematic LS testing can be incorporated into standard CRC pathways with minimal training required for existing teams to obtain consent for LS testing. There was a high uptake of LS testing among patients. Targeted testing for LS would have missed three quarters of cases, and by inference is a lost opportunity to discuss strategies to prevent cancer or detect cancer at an early stage with patients and their families.

\section{OUTCOME OF DIRECT ACCESS IBD PHYSICIAN DELIVERED ENDOSCOPY FOR GENERAL PRACTICE REFERRALS WITH SUSPECTED IBD}

Sohail Rahmany*, Matt Stammers, Louise Downey, Trevor Smith, Richard Felwick, Fraser Cummings, Markus Gwiggner. University Hospital Southampton NHS Foundation Trust, Southampton, UK

\subsection{6/gutjnl-2020-bsgcampus.69}

Introduction Patients with suspected IBD referred by primary care (GP) are traditionally seen in gastroenterology outpatient clinics followed by endoscopic investigations. This 2 phase model leads to delay in diagnosis and treatment, increasing pressure on gastroenterology outpatient services while still requiring endoscopic intervention. Our novel pilot project compared outcomes between direct-access IBD physician-delivered endoscopy versus the traditional clinic model for patients with suspected IBD.

Method A prospective cohort of consecutive patients referred by GP with suspected IBD were triaged either direct to IBD endoscopy $(n=50)$ or to outpatient IBD clinic followed by IBD endoscopy $(n=50)$ at the discretion of 10 gastroenterology consultants grading GP referrals. Data on demographics, faecal calprotectin, C-reactive protein, endoscopy outcomes, treatment, and follow up was collected. (Group $\mathrm{A}=$ direct to IBD endoscopy and Group B = IBD endoscopy via IBD clinic).

Results Both groups were age and gender-matched. Group A had a higher mean calprotectin (1363 ug/g vs $302 \mathrm{ug} / \mathrm{g}$ ) and a higher C-reactive protein $(10.6 \mathrm{mg} / \mathrm{l}$ vs $4.5 \mathrm{mg} / \mathrm{l})$. In Group A only $38 \%$ had a full colonoscopy versus $86 \%$ in Group B. Definitive diagnosis and treatment at time of IBD endoscopy took 27 days in Group A versus 212 days in Group B. Treatment with immunomodulators and biologics was similar in both groups but mesalazine and steroid use was higher in

\begin{tabular}{lll} 
Abstract 069 Table 1 & \multicolumn{2}{l}{ Diagnostic breakdown } \\
\hline & $\begin{array}{l}\text { Direct to endoscopy } \\
\text { (Group A) }\end{array}$ & $\begin{array}{l}\text { IBD endoscopy via IBD } \\
\text { clinic (Group B) }\end{array}$ \\
\hline Ulcerative colitis & $44 \%$ & $10 \%$ \\
Crohn's disease & $18 \%$ & $28 \%$ \\
IBDU & $8 \%$ & $4 \%$ \\
Diverticulosis/associated & $6 \%$ & $4 \%$ \\
segmental colitis & & \\
IBS & $24 \%$ & $50 \%$ \\
Bile sale malabsorption & $0 \%$ & $4 \%$ \\
\hline
\end{tabular}


Group A due to more severe disease and higher rate of ulcerative colitis, table 1 shows the diagnostic breakdowns from both groups following endoscopy. The IBD pick up was significantly higher in Group A with $70 \%$ vs 42\%. Endoscopy DNA rate was twice as high in Group B $(n=6)$. The direct to IBD endoscopy pathway resulted in 50 less initial IBD consultant clinics (100\% reduction) with a follow-up shift from IBD consultant to IBD nurse clinics.

Conclusion Triaging patients referred with suspected IBD directly to IBD physician delivered endoscopy resulted in more than a 26-week reduction in time to diagnosis and treatment while saving $100 \%$ of initial IBD consultant clinics. IBD pick up was high at $70 \%$ in direct to IBD endoscopy group, identifying a higher-need IBD population. Triaging GP referrals with suspected IBD direct to IBD endoscopy delivers rapid assessment and treatment.

\section{O70 PATIENT REPORTED EXPERIENCES THROUGH PBC FOUNDATION APP: WHAT IMPACTS UPON A PBC PATIENT'S EXPERIENCE?}

${ }^{1}$ Robert Mitchell-Thain*, ${ }^{2}$ Andrew Yeoman, ${ }^{3}$ Vinod Hegade. ${ }^{1} P B C$ Foundation, Edinburgh, UK; ${ }^{2}$ Hepatologist, Gwent Liver Unit, Newport, UK; ${ }^{3}$ Consultant Hepatologist, Leeds Liver Unit, Leeds, UK

\subsection{6/gutjnl-2020-bsgcampus.70}

Intro The $\mathrm{PBC}$ Foundation is a UK-based patient support organisation which supports patients with $\mathrm{PBC}$ in over 75 countries, affected by Primary Biliary Cholangitis (PBC). The PBC Foundation has developed a self-management App to conduct surveys to anonymously record Patient Recorded Outcome Measurements (PROMS) and Patient Recorded Experience Measures (PREMS). This App went live in 2019 with patients from 20 different countries registered and over 3200 downloads.

The aim of this study is to report the early aggregate findings of the first surveys conducted through the App and provide future directions on how this may be utilised in improving PBC patient care.

Methods In Nov 2019, we set two App-based surveys. The surveys have $n=189$ and $n=171$, respectively.

Results We found, firstly, that age has an impact upon the quality of care experienced by PBC patients. We asked patients, 'If, for any reason, you have not fully responded to Urso, have you been offered an additional second-line treatment?' The answer varied between age groups:

31-40 yrs, 31\% no: $41-50$ yrs, 47\% no: 51-60 yrs, 54\% no: $61-70$ yrs, $48 \%$ no: $70+y r s, 83 \%$ no, with an overall rate of $49.5 \%$ no.

We also asked, 'If, for any reason, you have not fully responded to Urso, has your clinician discussed second-line treatments with you?' Again, the answers were low at baseline, and correlated with patient age:

31-40 yrs, 27\% no: $41-50$ yrs, $38 \%$ no: $51-60$ yrs, $46 \%$ no: $61-70$ yrs, $45 \%$ no: $70+y r s, 50 \%$ no, with an overall rate of $41.5 \%$ no.

In terms of symptom management, one factor that appears to impact upon the patient experience is whether they see a gastroenterologist or a hepatologist. We asked, 'Has your clinician offered any advice or treatments to manage your fatigue. Interestingly, only $12.7 \%$ of patients experienced no fatigue. Of the $87.3 \%$ who did experience fatigue, only $17 \%$ of
Gastro patients and 23\% of Hep patients answered, 'yes.' When asked, 'Has your clinician offered any advice or treatment to manage your itch?' $67 \%$ of Gastro patients and $47 \%$ of Hep patients said yes.

Conclusions The data strongly suggests that there is significant unmet need, in terms of both disease management and symptom management, in PBC patients: particularly in those patients most in need of clinical intervention. The PBC Foundation App surveys provide useful insights into issues prevalent in the care of patients.

\section{INTRODUCING AN ELECTRONIC ALCOHOL SCREENING TOOL IMPROVES ALCOHOL MISUSE PATIENT IDENTIFICATION REDUCES LENGTH OF STAY}

Julius Mukarati*. Uhcw Nhs Trust, Coventry, UK

\subsection{6/gutjnl-2020-bsgcampus.71}

Introduction The Modified Single Alcohol Screening Questionnaire was a paper-based tool recorded in the Emergency Department booklet which was being under-utilised and difficult to audit. We had suspected that the length of stay was high in this patient group due to lack of identification resulting in missed opportunities to intervene effectively. Therefore we conducted an audit considering a consecutive random 100 patients retrospectively in 2017 based on the referral received showing that only $40-60 \%$ were being referred into the Alcohol Liaison Service thus we sought to improve the identification through the introduction of an electronic alcohol screening tool.

Method The electronic alcohol tool has an inbuilt alcohol assessment module on Vital Pac taking the nurse or clinician through a series of relevant alcohol questions in relation to the admission and derives at a score which determines the risk levels regarding that patient. The tool was implemented initially on a trial basis and fully implemented following staff training. The tool was then re-audited in 2019 reviewing electronic records stored on Vital Pac and our Clinical Results Reporting System (CRRS).

Results The results post implementation show an increase in compliancy which rose to $70-90 \%$ of patient being screened for alcohol. The number of referrals and patient contact also increased by approximately $7 \%$ as the patient contact rose from $47 \%$ in 2016 to $54 \%$ in 2019 , which, meant intervention was more timely therefore minimising risk. The total referrals in 2019 were 823 patients in comparison to 760 patients in 2018 showing a $4 \%$ increase in referrals due to the electronic tool prompting staff to refer patients identified as being at risk. The tool improved efficiency with robust data collection for audit. Bed days reduced per month from an average of $6-3$ bed days.

Conclusion The alcohol electronic screening reduced omissions and increased contact, minimising risk to patients. Cost effectiveness was noted through reduction in bed days due to effective patient management. The tool aided in promoting quality and safety in patient care delivery through, managing this patient group in a timely manner, improvement quality and suitable intervention, reducing risk and harm to this patient group, empowering clinical staff to recognise this patient group and be confident to manage their care effectively through knowledge and awareness as well as improve pathways for this patient group and trust policies. 